\title{
A plea for renin-angiotensin system blockers as first-line treatment in cases of severe acute hypertension
}

\author{
Sébastien Rubin (D) ${ }^{1,2}$, Romain Boulestreau ${ }^{3}$, Philippe Gosse ${ }^{4}$ and Christian Combe (D) ${ }^{1,5}$ \\ ${ }^{1}$ Néphrologie, Transplantation, Dialyse et Aphérèse, Hôpital Pellegrin, CHU de Bordeaux, Bordeaux, France, ${ }^{2}$ Unité INSERM U1034, Univ. \\ Bordeaux, Bordeaux, France, ${ }^{3}$ Cardiologie, Centre Hospitalier de Pau, Pau, France, ${ }^{4}$ Cardiologie et Hypertension artérielle, European \\ Hypertension Excellence Centre, Hôpital Saint André, CHU de Bordeaux, Bordeaux, France and ${ }^{5}$ Unité INSERM Biotis U1026, Univ. Bordeaux, \\ Bordeaux, France
}

Correspondence to: Christian Combe; E-mail: christian.combe@chu-bordeaux.fr

The management of hypertension as a chronic disease is now well codified. Over the last 10 years, blockers of the renin-angiotensin system (RAS) have become first-line drugs, often combined with diuretics or calcium channel blockers, particularly in the population of patients with chronic kidney disease [1]. The management of acute and severe hypertension is much less clear-cut. Expert recommendations in this area are based on very low levels of evidence. However, there is a relative consensus on treatment based on intravenous (IV) infusion of antihypertensive therapy in the first days. Labetalol, nicardipine and urapidil are the most frequently used drugs in this setting. The aim is to progressively reduce blood pressure (BP) to $140 / 90 \mathrm{mmHg}$ in the first 2 or 3 days. We believe that these recommendations result in underuse of RAS blockers [2].

It is necessary to differentiate between a severe initial presentation of chronic hypertension that does not require emergency treatment, but rapid assessment, and severe hypertension reflecting an acute disease that requires urgent treatmentaortic dissection, acute pulmonary oedema and stroke-induced hypertension should be managed according to the recommendations specific to these pathologies, which we will not discuss here [3]. All other severe hypertensive states requiring urgent treatment have in common small-vessel ischaemia: thrombotic microangiopathy (TMA)-induced hypertension; malignant hypertension, or better called as multiorgan damage hypertension [4]; diffuse microvascular injury [3]; scleroderma renal crisis (SRC); haemolytic uraemic syndrome; and thrombotic thrombocytopenic purpura. Within the kidneys, all of these conditions result in high renin secretion. Nearly 50 years ago, Bühler et al. [5] had already reported high renin secretion in cases of malignant hypertension. This supports the view that malignant hypertension is a high-renin hypertension secondary to TMA, triggers of which are unknown and/or probably multiple. Renal ischaemia-induced renin secretion rapidly produces a vicious circle that can exacerbate renal lesions and probably contributes to vascular lesions in other organs [6]. The RAS stimulates the sympathetic nervous system, which itself stimulates renin production via B1 renal receptors. Furthermore, pressureinduced natriuresis further triggers RAS activation.

Being convinced that renal ischaemia and renin activation are the cornerstone of the pathogenesis of malignant hypertension and given that previous series reported efficient and safe results with angiotensin-converting enzyme inhibitors (ACEis) in malignant hypertension [5], we have focused our research for $>20$ years in our centre on the treatment of malignant hypertension using only oral ACEis [7]. Our protocol involves early initiation of a small dose of an ACEi (for instance, ramipril $1.25 \mathrm{mg}$ ), after a saline infusion in cases of hypovolaemia, followed by a forced titration $(2.5 \mathrm{mg}$ at $6 \mathrm{~h}, 5 \mathrm{mg}$ at $12 \mathrm{~h}, 10 \mathrm{mg}$ at $24 \mathrm{~h}$ and a maximum of $10 \mathrm{mg}$ twice a day for the second day) if well tolerated, with a target systolic BP (SBP) of between 140 and $180 \mathrm{mmHg}$ in the first $48 \mathrm{~h}$, with close monitoring of BP and creatinine levels. When the maximum ramipril dosage is reached, a calcium channel blocker is added if the SBP is $>140 \mathrm{mmHg}$. The third drug class added is a thiazide diuretic, and if necessary, we finally introduce spironolactone at $25 \mathrm{mg}$. Beta blockers are introduced or continued if required by a specific indication (Table 1). Renal function and potassium levels are monitored daily. In all reports that we have published on our experience with this protocol, we have been challenged by reviewers who were not convinced by this strategy $[2,6,7]$. Three main concerns were raised: the risk of acute kidney injury (AKI); hyperkalaemia; and less effective BP control than with IV drug use.

Regarding the first two points, learning from the case of SRC (probably the most typical presentation of TMA-induced hypertension encountered by nephrologists) should convince us that raised creatinine levels is not the main concern in the treatment of severe hypertension. When the SBP reaches very high levels $(>230-250 \mathrm{mmHg})$, patients are at immediate risk of stroke, cardiac failure and death. If dialysis is necessary, it should not lead us to discontinue an indispensable treatment to break the vicious circle described above. Older reports of patients saved by bilateral nephrectomy before the availability 


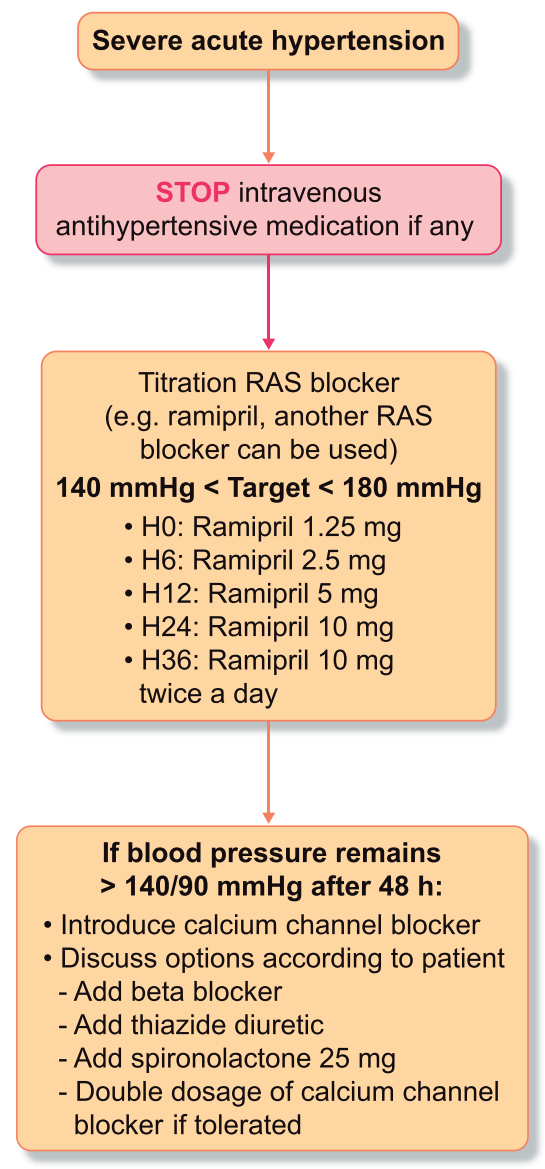

FIGURE 1: Treatment protocol used in our unit in cases of malignant hypertension. H0: admission, H6 and following numbers: hours after admission.

of RAS blockers should not be forgotten, albeit obviously coming from another era [8]. For SRC, ACEi use started in the 1980 s, resulting in increased 1-year survival from $15 \%$ to $75 \%$ not through better BP control, but mainly because intense activation of the RAS is very deleterious [9]. In a French cohort of SRC patients (2012), $>50 \%$ of the patients required dialysis, of whom more than half were able to be discharged within 2 years [10]. Despite the frequent need for dialysis, no nephrologists would question the use of RAS blockers as first-line treatment for the management of high BP in these patients. Moreover, severe AKI requiring dialysis and severe hyperkalaemia remain uncommon. In our series of malignant hypertension (baseline creatinine: $173 \pm 165 \mu \mathrm{mol} / \mathrm{L} ; n=153$ ), no patients required dialysis during the acute phase, and because of secondary hyperaldosteronism with baseline potassium levels being low $(3.5 \pm 0.6 \mathrm{mmol} / \mathrm{L})$, none of the patients had potassium levels $>6 \mathrm{mmol} / \mathrm{L}$ that required ACEi discontinuation during the acute phase follow-up [7].

Regarding the third concern, oral management is efficient. In our series, at discharge, the SBP decreased from $211 \pm 33$ to $141 \pm 15 \mathrm{mmHg}(\mathrm{P}<0.001)$ and we did not observe any cerebral haemorrhage during the acute phase. Moreover, the use of IV therapeutics is not beyond reproach-in the Studying the
Treatment of Acute hyperTension (STAT) study, the IV strategy applied to patients with a hypertensive emergency was associated with $5.6 \%$ of patients with sudden hypotension requiring intervention and $33 \%$ of patients with recurrent acute hypertension [11]. These adverse events were reported to be associated with increased mortality [12].

Last but not the least, using RAS blockers is less expensive than IV antihypertensive treatment and can be used more easily in low- and middle-income countries where malignant hypertension occurs more frequently and use of IV therapy is usually not an option.

We consider it essential to extend the use of RAS blockers as the first line of treatment in cases of severe hypertension requiring urgent treatment. This is in line with the pathogenesis of these hypertensions and is evidenced by the effectiveness of our therapeutic strategy. We need to generalize the lessons learnt from the management of SRC, so that we use RAS blockers in acute hypertensive emergencies.

\section{CONFLICT OF INTEREST STATEMENT}

None of the authors have any conflict of interest to declare related to the content of this article.

\section{REFERENCES}

1. Williams B, Mancia G, Spiering W et al.; ESC Scientific Document Group. 2018 ESC/ESH Guidelines for the management of arterial hypertension. Eur Heart J 2018; 39: 3021-3104

2. Rubin S, Combe C, Gosse P. Acute severe hypertension. N Engl J Med 2020; 382: e11

3. Peixoto AJ. Acute severe hypertension. N Engl J Med 2019; 381: 1843-1852

4. Cremer A, Amraoui F, Lip GYH et al. From malignant hypertension to hypertension-MOD: a modern definition for an old but still dangerous emergency. J Hum Hypertens 2016; 30: 463-466

5. Bühler FR, Laragh JH, Vaughan ED et al. Antihypertensive action of propranolol. Specific antirenin responses in high and normal renin forms of essential, renal, renovascular and malignant hypertension. Am J Cardiol 1973; 32: 511-522

6. Gosse P, Boulestreau R, Brockers C et al. The pharmacological management of malignant hypertension. J Hypertens 2020; 38: 2325-2330

7. Rubin S, Cremer A, Boulestreau R et al. Malignant hypertension: diagnosis, treatment and prognosis with experience from the Bordeaux cohort. $J$ Hypertens 2019; 37: 316-324

8. Cruz I, Callender CO, Cummings Y et al. Malignant hypertension revisited: the role of bilateral nephrectomy. J Natl Med Assoc 1980; 72: 453-457

9. Steen VD, Costantino JP, Shapiro AP et al. Outcome of renal crisis in systemic sclerosis: relation to availability of angiotensin converting enzyme (ACE) inhibitors. Ann Intern Med 1990; 113: 352-357

10. Guillevin L, Berezne A, Seror R et al. Scleroderma renal crisis: a retrospective multicentre study on 91 patients and 427 controls. Rheumatology (Oxford) 2012; 51: 460-467

11. Katz JN, Gore JM, Amin A et al. Practice patterns, outcomes, and endorgan dysfunction for patients with acute severe hypertension: the Studying the Treatment of Acute hyperTension (STAT) registry. Am Heart J 2009; 158: 599-606.e1

12. Mayer SA, Kurtz P, Wyman A et al. Clinical practices, complications, and mortality in neurological patients with acute severe hypertension: The Studying the Treatment of Acute hyperTension registry. Crit Care Med 2011; 39: 2330-2336

Received: 13.10.2020; Editorial decision: 14.10.2020 